\title{
Improvement of leak tightness for swellable elastomeric seals through the shape optimisation
}

Y. Gorash

Department of Mechanical \& Aerospace Engineering, University of Strathclyde, Glasgow G1 1XJ, UK

A. Bickley

Weir Advanced Research Centre, Weir Group, Technology \& Innovation Centre, Glasgow G1 1RD, UK

F. Gozalo

Weir Minerals, Weir Rubber Engineering, Weir Group, Salt Lake City, UT 84119, USA.

\begin{abstract}
Swellable packers have been widely employed in various oil \& gas applications. Downhole conditions are difficult to reproduce using physical testing environments, but can be simulated in a virtual environment using CAE software. A better understanding of packers' mechanical behaviour in downhole conditions would provide a higher confidence and improvement in existing engineering design practices for the manufacturing of packers. The numerical simulation can be incorporated into optimisation procedures searching for an optimal shape of packers aiming to minimise the time to seal the borehole and maximise the contact pressure between the seal and borehole. Such an optimisation would facilitate the development of a packer with various designs optimised for different downhole conditions. The objective of this work is to develop a design tool integrated into Abaqus/CAE to implement parametric numerical studies using implicit and explicit FE-simulations. However, development of such a CAE plugin is associated with a number of technical challenges specific to this class of multiphysics problems, which are addressed in this research and discussed in the paper.
\end{abstract}

\section{INTRODUCTION}

Swellable elastomers are a special type of polymer with the ability to swell when exposed to water or oil. They are used in production of swellable elastomeric seals, a type of specifically engineered packer that activates upon contact with wellbore fluids. Such packers have been widely employed in various oil \& gas applications including slimming of well design, zonal isolation, water shut-off, and multi-stage fracturing. Referring to Lou \& Chester (2014), downhole service conditions are extremely challenging to reproduce using physical testing environments, but can be simulated in a virtual environment using software for multiphysics engineering analysis. A better understanding of packers' mechanical behaviour in downhole conditions in a virtual environment would provide a higher confidence and improvement in existing engineering design practices for manufacturing of packers, as demonstrated e.g. by Akhtar, Qamar, Pervez, \& Al-Jahwari (2013).

The service characteristics of packers, which are generally measured from full-scale packer tests, can be predicted through numerical FE-simulations based on material data obtained from basic mechanical experiments. The experiments required for comprehensive material input would focus on evolution of hyperelastic properties (in tension, compression and shear) with the change of volume / density of specimens during swelling. The numerical simulation of packers can be incorporated into optimisation procedures finding an optimal shape of packers aiming to minimise the time to seal the borehole and maximise the contact pressure between the seal and borehole (Lou \& Chester 2014). Such an optimisation procedure would facilitate the development of a packer with various designs optimised for different downhole conditions considering the borehole type, size and temperature. The objective of this research project is to develop a design tool integrated into Abaqus/CAE to implement parametric numerical studies using advanced FE-simulation to provide an improved design of packers for various downhole conditions. However, the implementation of the packer swelling simulation is associated with a number of technical challenges specific to this particular class of multiphysics problems, which are illustrated in Fig. 1 and listed below: 


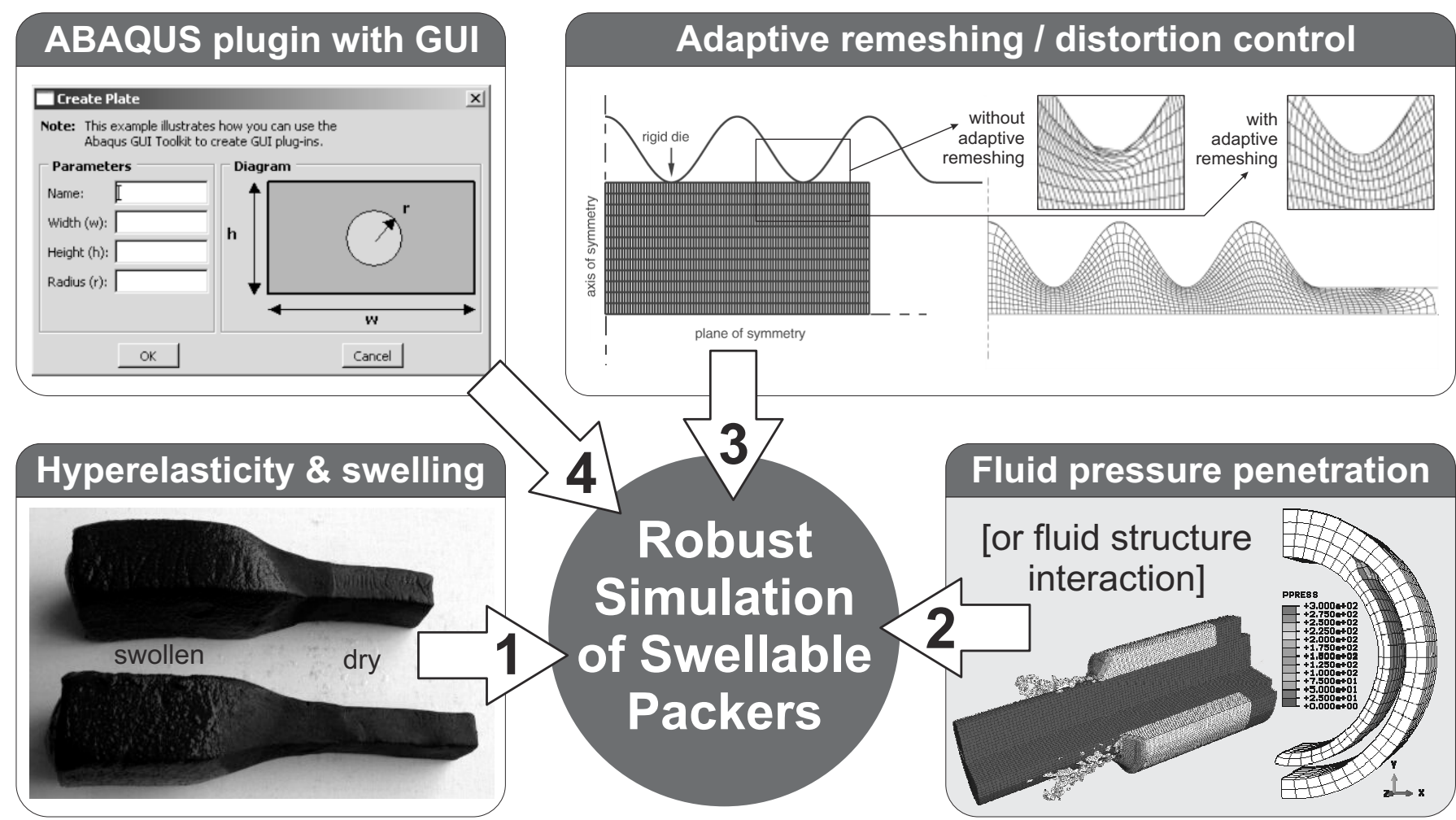

Figure 1: Diagram of technical requirements for a robust FE-simulation of swellable packers for progressive failure and leakage

1. The key component is an advanced material model comprising both hyperelasticity and moisture swelling. It has to consider two-way interaction between mechanical response and swelling capacity. Implementation of such a material model requires programming of a Fortran subroutine for the user defined material using the Flory \& Rehner Jr. (1943) theoretical background, which is presented in the first instance by Flory-Rehner equation:

$-\left[\ln \left(1-\nu_{2}\right)+\nu_{2}+\chi_{1} \nu_{2}^{2}\right]=V_{1} n\left(\nu_{2}^{\frac{1}{3}}-\frac{\nu_{2}}{2}\right)$

where $\nu_{2}$ is the volume fraction of polymer in the swollen mass, $V_{1}$ the molar volume of the solvent, $n$ is the number of network chain segments bounded on both ends by cross-links, and $\chi_{1}$ is the Flory solventpolymer interaction term.

In polymer science Eq. (1) describes the mixing of polymer and liquid molecules as predicted by the equilibrium swelling theory of Flory \& Rehner Jr. (1943). It describes the equilibrium swelling of a lightly cross-linked polymer in terms of crosslink density and the quality of the solvent. The theory considers forces arising from three sources:

- the entropy change caused by mixing of polymer and solvent;

- the entropy change caused by reduction in number of possible chain conformations via swelling;

- the heat of mixing of polymer and solvent, which may be positive, negative, or zero.
2. The moisture swelling process is not uniform and starts on the surfaces which are subject to fluid. Adsorption, which governs the progress of swelling can occur only at free surfaces. Therefore, the fluid pressure penetration needs to be incorporated into the simulation (Simulia [PPL] 2016) and directly linked to swelling. Distributed pressure penetration load allows for the simulation of fluid penetrating into the surface between two contacting bodies, penetration of fluid from multiple locations on the surface, and application of the fluid pressure normal to the surfaces. It automatically adjusts the application of a fluid pressure depending on changes of contact conditions.

3. Non-uniform swelling is associated with a localised increase of material volume. It may cause a significant distortion of FE mesh and arouse FEA convergence problems. To overcome this, there are a few options available in the setup of the FE-model (Simulia [AT] 2016) including a mesh-to-mesh solution mapping (Abaqus/Standard), adaptive remeshing (Abaqus/Explicit) and element distortion control.

4. Parametric study assumes considering a large number of different geometric configurations, looking at material properties variation and different downhole conditions. Basically this means a search for an optimal geometry through a sensitivity study, which would result in specific design recommendations for the geometry of a packer. Therefore, it would be reasonable to automate the analysis procedure through an Abaqus plug-in (Puri 2011) with a convenient graphical user interface (GUI), which provides access to the parameters of geometry, material properties and service conditions. 


\section{SHAPE OPTIMISATION}

Over recent years, non-parametric optimisation established as standard methods to improve the overall design and reliability of structural components under extreme loading conditions. The purpose of nonparametric optimization is to give engineers a method to define a design space in regions or whole components without the process of defining the problem in design parameters (Brieger 2016). Much more freedom in terms of possible design modification is a clear advantage of non-parametric methods (presented by topological and shape optimisation), when compared to parametric optimisation.

In relation to swellable packers, their principal dimensions (diameter and length) can be optimised parametrically, while the external contact surface can be optimised non-parametrically for better sealing capability considering its contact with the borehole. But non-parametric optimisation of structures under contact conditions is a quite specific problem because of its non-smooth character, which is not extensively studied. However, Wagner \& Helfrich (2016) revealed great potential of the topology and shape optimization under contact conditions using examples from literature and industry. Various constraints, such as contact pressure, compliance and stresses can be used as objective functions for this class of problems. In addition, release directions, symmetry conditions and frozen (or fixed) regions can be considered as side constraints of the optimization process with limits in terms of weight and volume. In contrast to Wagner \& Helfrich (2016) who performed optimisation with industrial codes (PERMAS and VisPER), in this work Simulia Tosca Structure is used. This is a more widely accessible software system for non-parametric structural optimisation that provides topology, sizing, shape and bead optimisation using industry standard FE-solvers (ANSYS, Abaqus, MSC Nastran).

A special usage of the shape controller algorithm in Simulia Tosca Structure is the optimization of contact zones, because small changes in the contact surface usually have a big influence on the contact pressure. Contact pressure can be either minimised as explained by Simulia [MCP] (2016), or maximised as needed in this work. Therefore, shape optimisation is used here to improve the grip of a packer with the surface of a borehole. The normal shape optimization stimulates the surface growth in contact zones, which results in a higher contact pressure and shrinkage in a lower.

For a test shape optimisation study, the trimmed version of a packer geometry from Lou \& Chester (2014) was used as benchmark problem with $L$ reduced from 16" to 2". The optimisation analysis resulted in a rippled external surface of a packer as shown in Fig. 2a with comparison to the original rectangular profile. The distribution of contact pressure became very non-uniform as shown in Fig. $2 b$ with four maximums which are about 5 times higher than
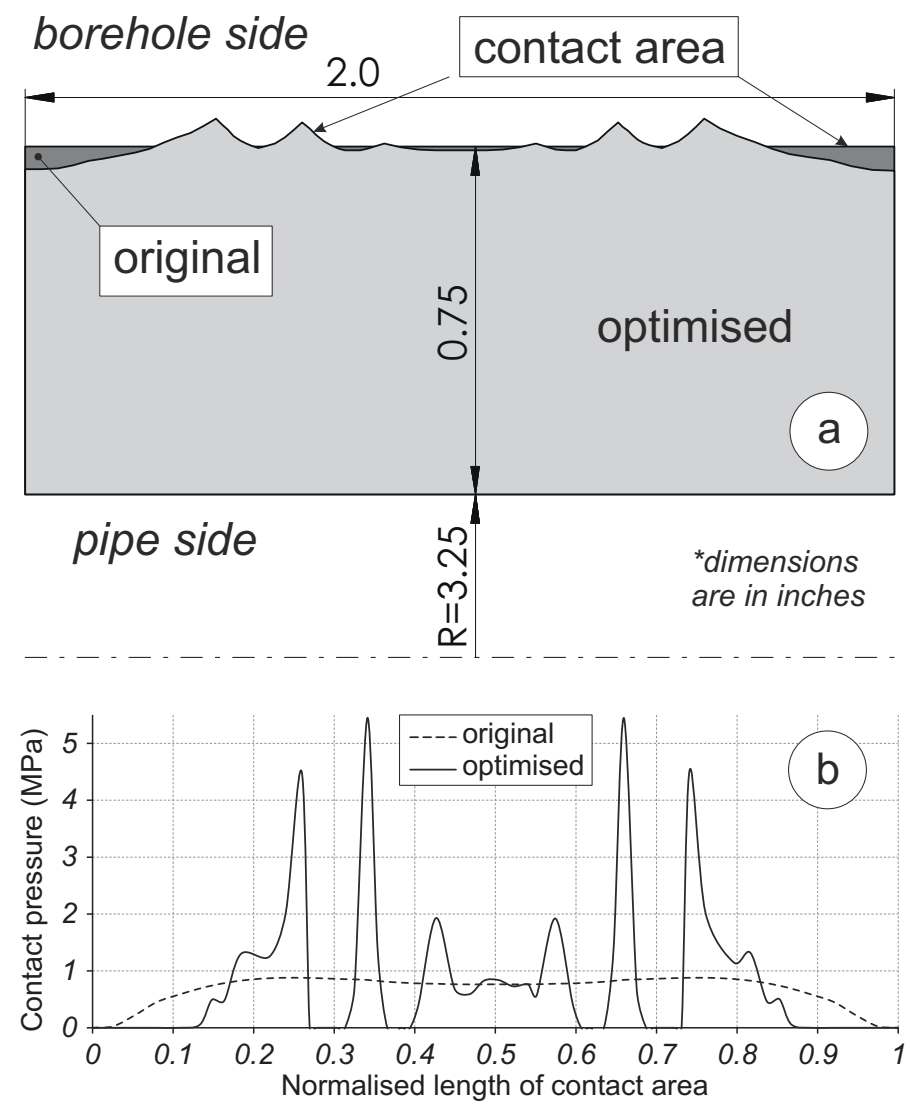

Figure 2: Packer profile shape optimisation with Tosca Structure: a) change of profile geometry, b) change of contact pressure

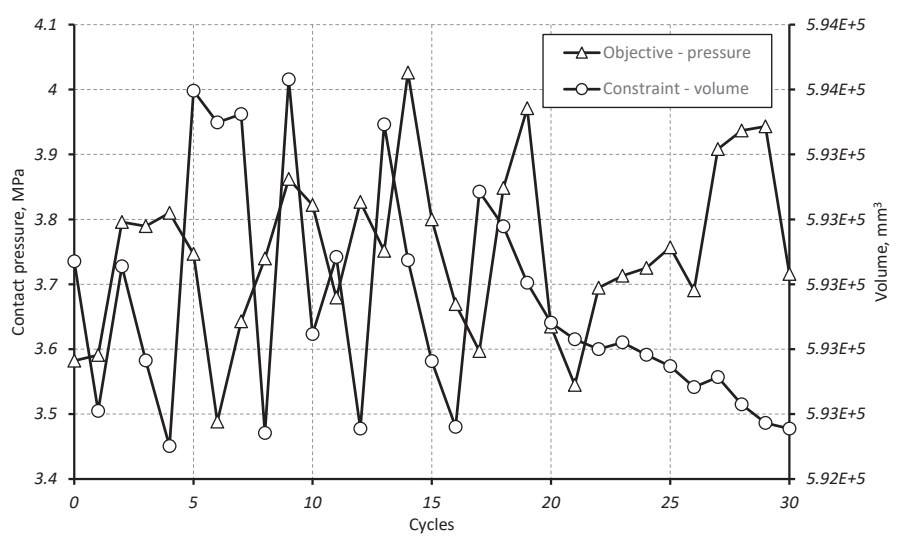

Figure 3: Convergence of the shape optimisation analysis with objective function and constraint vs time

the original smooth contact pressure. The final result presented in Fig. 2 has been obtained after 30 iterations when a target convergence was achieved as illustrated in Fig. 3 comparing the evolution of objective (contact pressure) to the variation of constraint (volume), which had to remain constant. The external surface was completely free to evolve considering free movement of nodes within the packer volume with a few geometric restrictions including 1) mirror symmetry; 2) packer sides remain flat; 3 ) demold control considering pull direction.

An important and final part of optimisation analysis is a validation of the obtained design, which in this study is expressed in terms of comparative sealing capability. The first attempt at validation analysis was performed using quasistatic simulation in Abaqus/Standard (Gorash, Bickley, \& Gozalo 2016). 


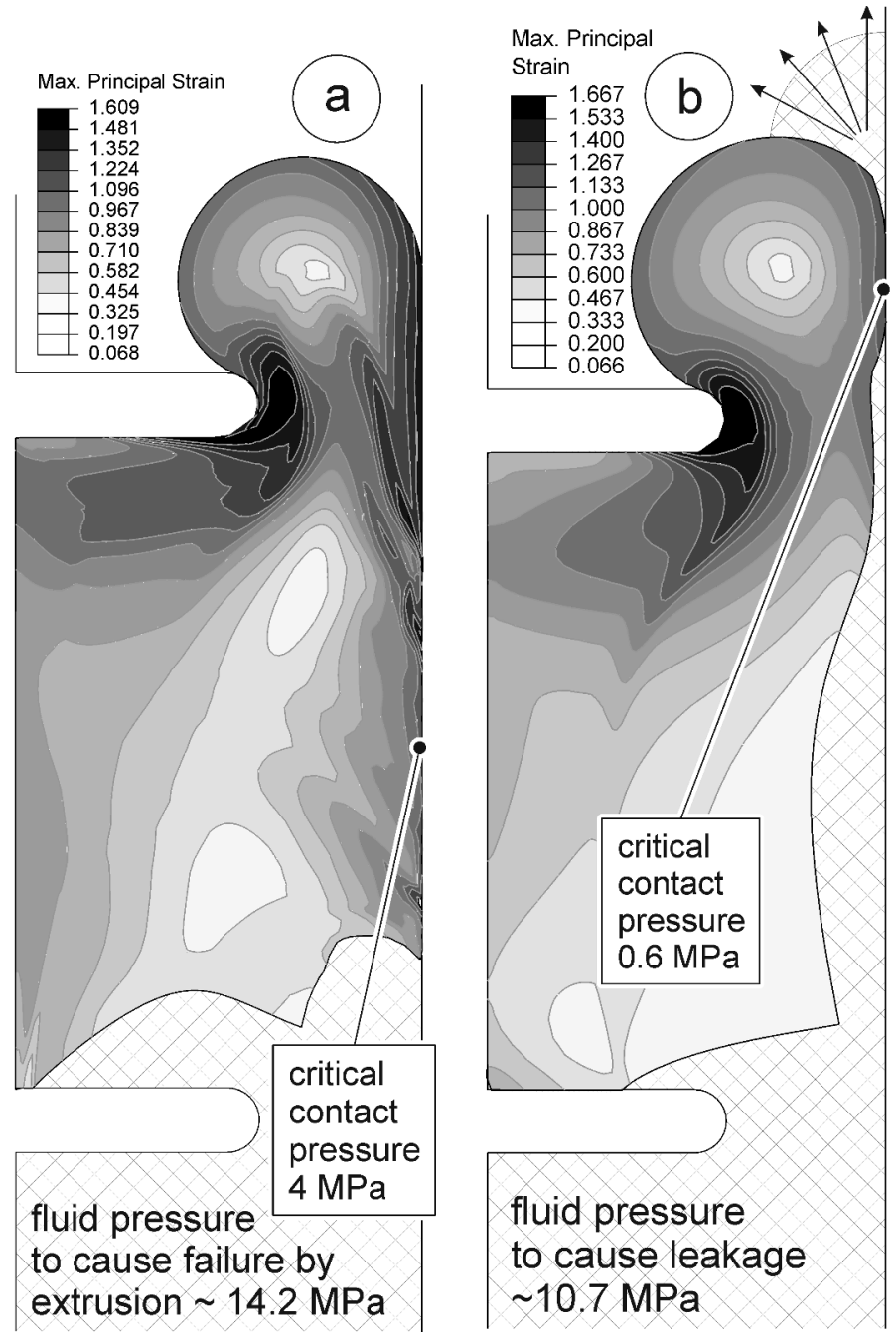

Figure 4: Validation simulation of the benchmark packer using (a) optimised and (b) original geometry with Abaqus/Standard

From obtained results, it was confirmed that the original flat surface packer can't seal effectively, because it allows the fluid to penetrate into the contact and eventually to separate the packer from the borehole, as shown in Fig. 4b. The sealing capability is better for the rippled surface packer, which doesn't let the fluid pressure penetrate at all, because of a stronger grip, as shown in Fig. 4a. This can be considered as a basic and qualitative validation of the optimisation result, which is sufficient to get an idea of the challenges and level of complexity associated with realistic simulation of swell packers. For a more clear, quantitative and comprehensive validation of the packer design, the simulation capabilities of Abaqus/Standard solver are insufficient.

\section{VALIDATION SIMULATION}

The validation simulation with Abaqus/Standard fails when trying to push the packer harder and to extrude it from the protective rings by applying excessive pressure. So there is no way to compare the maximum applicable pressure for original and optimised designs, because the maximum pressure for an optimised design is not achievable. The quasistatic analysis fails to converge because of excessive distortion of elements attributed to the seal, which stick to the ring, and borehole surfaces. Automatic adaptive remeshing is not available as a part of Abaqus/Standard functionality, therefore extrusion problems with extreme deformation can't be effectively solved using this product. But the good thing about Abaqus/Standard is the availability of pressure penetration load (PPL) explained in Simulia [PPL] (2016). This functionality replaces the computationally expensive fluid structure interaction, when the structural analysis is in focus. In application to this work, PPL allows to consider for a leakage probability, when it occurs through the contact surface without excessive extrusion.

Therefore, following the presentation of Gorash, Bickley, \& Gozalo (2016) and subsequent discussion, it was decided to switch to the more advanced solver - Abaqus/Explicit, which is recognised as a more robust solver when it comes to very non-linear problems and extremely large deformations, which can be experienced by packer under very high pressure. The validation simulation using PPL for a full-size packer geometry from Lou \& Chester (2014), not a benchmark, can't be completed when fluid pressure approaches a full penetration through the contact surface, because ABAQUS/Standard solver fails to converge with errors related to excessive distortion of elements caused obviously by extrusion. It would have taken a vast amount of efforts to extend the functionality of Abaqus/Standard to a suitable level to proceed with validation. The first drawback of Abaqus/Standard solver with excessive element distortion could be fixed by application of the mesh-to-mesh solution mapping (Simulia [AT] 2016), but it is not automatic. In order to automate the meshto-mesh solution mapping, customisation via Python script would be required. The second drawback of Abaqus/Standard solver with the fluid pressure only penetrating (available as standard feature), is that it would require a customisation via FORTRAN subroutine to enable the receding of the fluid pressure from the closed contact surfaces.

Abaqus/Explicit is a special-purpose solver that employs an explicit time integration scheme to solve highly non-linear systems with many complex contacts under transient loads, which is appropriate in many dynamic applications, such as drop tests, crushing, manufacturing processes and hydraulic fracturing. Abaqus/Explicit is more computationally expensive compared to Abaqus/Standard, but this obstacle is possible to overcome by running simulations on an HPC facility for simulations with big size models. This solver significantly expands the progressive failure analysis capabilities, and actually eliminates any limitations related to non-linearities, large deformations and transient / dynamic effects. The best prove of its efficiency is a solution of a so-called pressfit problem (Wriggers 2006), when a cylindrical rubber block compressed from the tube of bigger diameter into the tube with a smaller diameter. In previous 


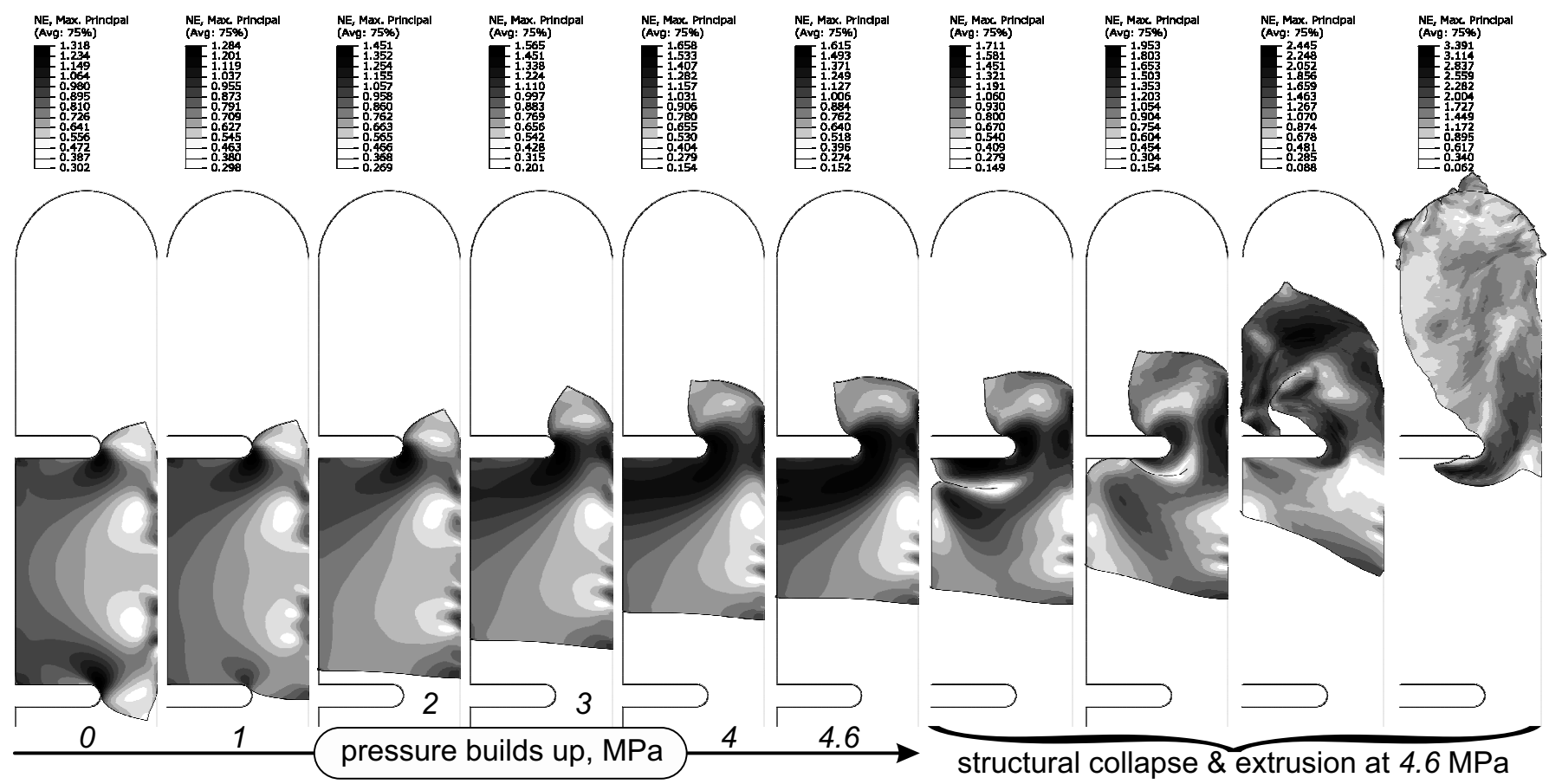

Figure 5: Validation simulation of the benchmark packer failure using optimised geometry with Abaqus/Explicit

work by Connolly, Gorash, \& Bickley (2016), a simple and stable solution for such a benchmark problem using standard solvers in Ansys and Abaqus couldn't be obtained in order to develop a robust approach to simulation. It should be noted that successful simulation became possible due to modification of the friction model used in analysis from the linear Coulomb to the bi-linear Coulomb-Orowan law (Raous 1999) expressed in terms of friction force as

$F_{f}=\min \left(\mu\left|F_{n}\right|, F_{\tau}\right)$,

where $\mu$ is a coefficient of friction, $F_{n}$ is a normal force, and $F_{\tau}$ is a critical share force, which corresponds to a critical shear stress $\tau_{c}$ in the FEA setup. The Coulomb term $\mu\left|F_{n}\right|$ is linear and describes the partial slip. When the critical value of $\tau_{c}$ is reached, the total slip occurs, which plays a key role in simulation convergence, because it prevents the rubber material from sticking to the rigid walls.

Therefore, the recent work focused on a development of practical approach to simulations of packers with Abaqus/Explicit, since the setup of analyses in Standard and Explicit solvers is quite different. The biggest advantages attributed to Explicit solver are automatic adaptive remeshing (in application to large plastic deformations) or distortion control of elements (in application to large hyperelastic deformations) and stable solution of contact problems with large relative displacements. Considering the superior robustness of Abaqus/Explicit, it is a minor drawback that PPL functionality is unavailable for Explicit analysis. The robustness of extrusion failure simulations for packers can be demonstrated on advanced validation analysis of benchmark problem. Since PPL is unavailable, the pressure is applied to the bottom surface and ramped in the course of simulation for both benchmark packers - original (with smooth surface) and optimised (with a rippled surface). Comparison of simulation results shows that the optimised packer (see Fig. 5) can bear about $10 \%$ of more pressure compared to the packer with a smooth surface. Due to application of Coulomb-Orowan friction law (2), the whole simulation splits into two parts - slow and gradual pressure build-up and very quick failure by extrusion when reaching a critical pressure of 4.6 $\mathrm{MPa}$. This can be considered as an additional validation of the optimisation result, which is extended with the comprehensive extrusion simulation, that was not possible with the Standard solver.

The validation simulation of a full-size real packer (Lou \& Chester 2014) in Fig. 6 demonstrates a complete extrusion of the packer. It also shows that extrusion is not gradual, it is rather abrupt with a distinctive critical pressure $(8.6 \mathrm{MPa})$ when sticking to protective rings can't stop progressive slipping any more.

\section{CONCLUSIONS}

It should be noted that the conducted validation simulations can lack realism, because interaction with fluid was not considered, which might result in a leakage through the contact surface and less severe extrusion. This simulation challenge can be effectively addressed by application of the Coupled Eulerian-Lagrangian (CEL) approach in Abaqus/Explicit which provides engineers with the ability to simulate a class of problems where the interaction between structures and fluids is important. This capability does not rely on the coupling of multiple software products, but instead solves the fluid-structure interaction (FSI) simultaneously within Abaqus. There is a great potential in CEL ap- 


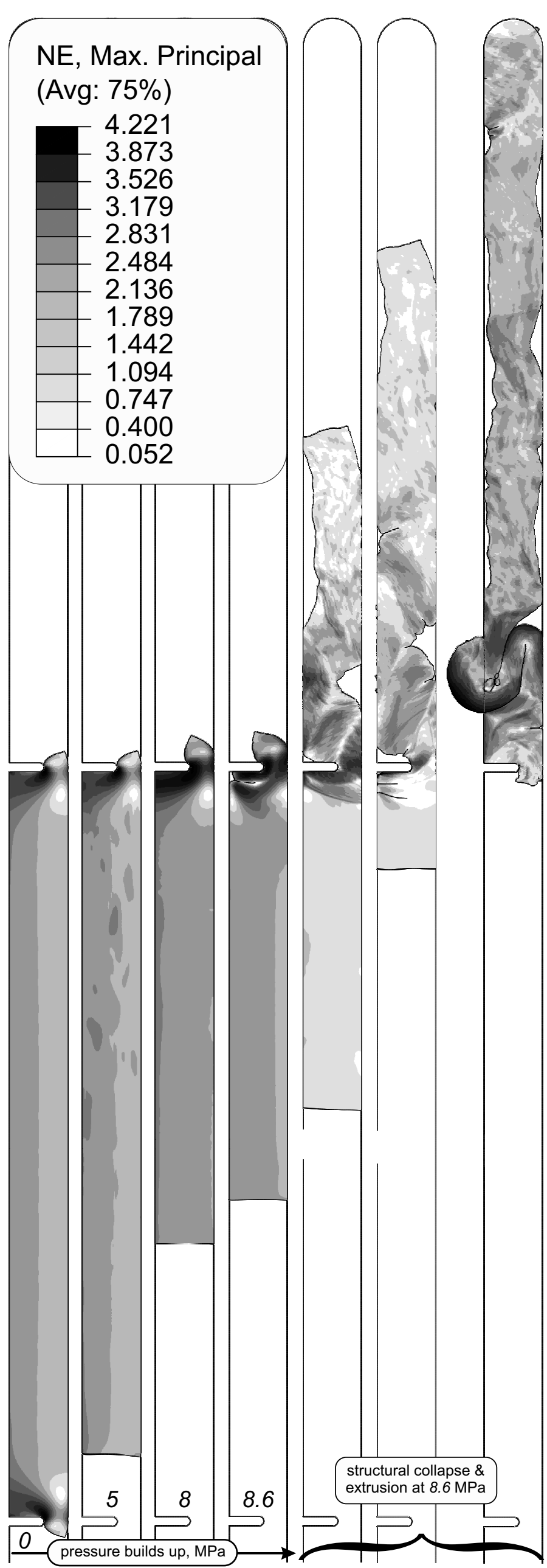

Figure 6: Validation simulation of the full-size packer failure using optimised geometry with Abaqus/Explicit proach for packers' leakage simulation. The highest level of realism in simulation of leakage process can be achieved engaging ABAQUS/Explicit and CEL this is where the future work will be focused.

\section{ACKNOWLEDGEMENTS}

The authors greatly appreciate the financial and material support of Weir Group PLC given within the WARC project "Design Optimisation of Swell Packers" and the University of Strathclyde for hosting during the course of this work. Results were obtained using the EPSRC funded ARCHIE-WeSt High Performance Computer (www.archie-west.ac.uk) - EPSRC grant no. EP/K000586/1.

\section{REFERENCES}

Akhtar, M., S. Z. Qamar, T. Pervez, \& F. K. Al-Jahwari (2013). FEM simulation of swelling elastomer seals in downhole applications. In Proc. ASME 2013 Int. Mechanical Engineering Congress \& Exposition (15-21 Nov 2013), Number IMECE2013-64312, San Diego, California, USA, pp. 1-7.

Brieger, S. (2016). Non-parametric optimization. In R. Steinbuch and S. Gekeler (Eds.), Bionic Optimization in Structural Design: Stochastically Based Methods to Improve the Performance of Parts and Assemblies, Section 2.6, pp. 3742. Berlin, Heidelberg: Springer.

Connolly, S., Y. Gorash, \& A. Bickley (2016). A comparative study of simulated and experimental results for an extruding elastomeric component. In 23rd Int. Conf. on Fluid Sealing 2016 (2-3 Mar 2016), Manchester: BHR Group, pp. 31-41.

Flory, P. J. \& J. Rehner Jr. (1943). Statistical mechanics of crosslinked polymer networks ii. swelling. The Journal of Chemical Physics 11(11), 521-526.

Gorash, Y., A. Bickley, \& F. Gozalo (2016). Design optimisation of swellable elastomeric seals using advanced material modelling and FEM simulations. In Poster - Int. Conf. on Innovations in Rubber Design (7-8 Dec 2016), London: IOM3.

Lou, Y. \& S. Chester (2014). Kinetics of swellable packers under downhole conditions. Int. J. Appl. Mechanics 06(06), 1450073 [18p].

Simulia [AT] (2016). ABAQUS Analysis User's Guide - 12.1.1 Adaptivity techniques (Version 2016 ed.). Providence, RI, USA: Dassault Systèmes Simulia Corp.

Simulia [MCP] (2016). Tosca Structure 2016 Documentation Minimizing contact pressure (Version 2016 ed.). Karlsruhe, Germany: Dassault Systèmes Simulia Corp.

Simulia [PPL] (2016). ABAQUS Analysis User's Guide - 37.1.7 Pressure penetration loading (Version 2016 ed.). Providence, RI, USA: Dassault Systèmes Simulia Corp.

Puri, G. (2011). Python Scripts for Abaqus: Learn by Example. USA: Kan sasana Printer.

Raous, M. (1999). Quasistatic signorini problem with Coulomb friction and coupling to adhesion. In P. Wriggers and P. Panatiotopoulos (Eds.), New Developments in Contact Problems, Number 388 in CISM International Centre for Mechanical Sciences, Chapter 3, pp. 101-178. Vienna: Springer-Verlag.

Wagner, N. \& R. Helfrich (2016). Topology and shape optimization of structures under contact conditions. In Proc. 1st Euro. Conf: Simulation-Based Optimisation (12-13 Oct 2016), Manchester, UK, pp. 127-130.

Wriggers, P. (2006). Discretization, large deformation contact. In Computational Contact Mechanics, pp. 225-307. Berlin, Heidelberg: Springer. 